\title{
Ethanolamine Plasmalogen Suppresses Apoptosis in Human Intestinal Tract Cells in Vitro by Attenuating Induced Inflammatory Stress
}

Ephantus Nguma, Shinji Yamashita,* Kei Kumagai, Yurika Otoki, Ayaka Yamamoto, Takahiro Eitsuka, Kiyotaka Nakagawa, Teruo Miyazawa, and Mikio Kinoshita

Cite This: ACS Omega 2021, 6, 3140-3148

Read Online

ABSTRACT: Ethanolamine plasmalogen (PlsEtn) is a subtype of ethanolamine glycerophospholipids (EtnGpl). Recently, PlsEtn has attracted increasing research interest due to its beneficial effects in health and disease; however, its functional role in colonic health has not been well established. This study was conducted to determine the mechanism underlying the antiapoptotic effect of PlsEtn in human intestinal tract cells under induced inflammatory stress. Lipopolysaccharide induced apoptosis of differentiated Caco- 2 cells, which was suppressed by EtnGpl in a dose-dependent manner. Cells treated with ascidian muscle EtnGpl containing high levels of PlsEtn demonstrated a lower degree of apoptosis, and downregulated TNF- $\alpha$ and apoptosis-related proteins compared to those treated with porcine liver

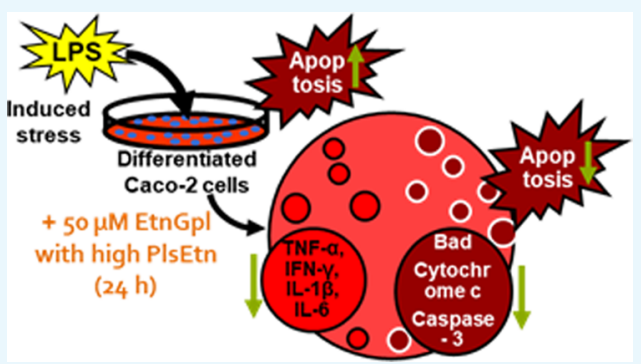
EtnGpl containing low PlsEtn. This indicates that PlsEtn exerted the observed effects, which provided protection against induced inflammatory stress. Overall, our results suggest that PlsEtn with abundant vinyl ether linkages is potentially beneficial in preventing the initiation of inflammatory bowel disease and colon cancer.

\section{INTRODUCTION}

Ethanolamine plasmalogen (PlsEtn), a subclass of ethanolamine glycerophospholipids (EtnGpl) is a universal phospholipid in mammalian membranes. PlsEtn contains a vinyl-ether (alkenyl) linkage at the $s n-1$ position, whereas at the $s n-2$ position, it is enriched with polyunsaturated fatty acids (PUFA). ${ }^{1,2}$ Marine sources such as sea squirt and mussel muscles are enriched with n-3 PUFA, notably docosahexaenoic acid (DHA, 22: 6n-3) and eicosapentaenoic acid (EPA, 20:5n3 ), while land sources such as pig and cattle muscles are enriched with n-6 PUFA arachidonic acid (ARA, 20:4n-6) at the $s n-2$ position. ${ }^{3,4}$ The vinyl ether linkage in PlsEtn has high oxidative potential, which enables the scavenging of free radicals and singlet oxygen, in addition to altering membrane properties. $^{1,2}$ Increased cellular levels of PlsEtn bearing n-3 PUFA have shown potential for the prevention of neurodegenerative disorders like Alzheimer's disease. ${ }^{5}$ It has also been reported that PlsEtn is involved in the development of other diseases such as Parkinson's disease and atherosclerosis. $^{6,7}$ Consequently, several researchers are currently interested in the association between colon carcinogenesis and PlsEtn levels.

Currently, colon cancer is a major health problem in both developed and developing nations. Worldwide, colon cancer ranks third in incidence and fourth in mortality rates among cancers. ${ }^{8}$ Chronic colon inflammation has been associated with an increased inflammatory bowel disease (IBD), which is a risk of colon cancer initiation and promotion. ${ }^{9}$ Chronic inflamma- tion is associated with excessive colon cell death (apoptosis), and on the other hand, disturbed regulation of colon cell death, leading to uncontrolled growth of cells (resistance to apoptosis), contributes to colon cancer promotion. ${ }^{10,11}$ Patients with chronic colon inflammation exhibit increased levels of pro-inflammatory cytokines such as interleukin (IL)-6, IL- $1 \beta$, IL- 8 , and tumor necrosis factor-alpha (TNF- $\alpha){ }^{12}$ This inflammatory microenvironment facilitates crosstalk with infiltrating immune cells to create a pro-carcinogenic environment. $^{11}$

It is well established that several endogenous regulatory molecules play a key role in regulating intestinal homeostasis, but their presence is significantly influenced by dietary compounds. ${ }^{11}$ Dietary compounds come into direct contact with the colonic epithelium cells and may affect growth, differentiation, and cell death within the tissue. ${ }^{13}$ In the recent past, research interest in the benefits of PlsEtn in health and disease has increased; ${ }^{1,14}$ however, its functional role in colonic health has not been well established. In our previous study, we observed that diet with high PlsEtn levels more efficiently

Received: November 12, 2020

Accepted: January 13, 2021

Published: January 22, 2021 
suppressed aberrant crypt foci (ACF) formation and apoptosis compared to the diet with low PlsEtn levels. The observed suppression was a consequence of higher downregulation of the pro-inflammatory mediator TNF- $\alpha$ and oxidative stress in 1,2-dimethylhydrazine(DMH)-treated mice. ${ }^{15}$ This suggests that the abundance of dietary PlsEtn altered the colon epithelial microenvironment and conferred a low susceptibility to carcinogenesis.

Based on our previous study, the aim of this study was to clarify the functional role of PlsEtn and the molecular mechanisms underlying its effects on colonic health. In this study, we investigated the effects of extrinsic PlsEtn from the ascidian muscle and porcine liver EtnGpl on inflammatory stressed differentiated Caco-2 cells as a human intestinal tract in vitro model and the possible underlying mechanisms. Extrinsic EtnGpl from the ascidian (Holacynthia roretzi) muscle contained higher levels of PlsEtn, EPA, and DHA, while EtnGpl from the porcine liver contained low levels of PlsEtn and high ARA.

\section{RESULTS}

2.1. EtnGpl Fraction from Ascidian Muscle and Porcine Liver. The level of PlsEtn in the ascidian muscle was $87.3 \mathrm{~mol} \%$ EtnGpl. The prominent acyl carbon chains in ascidian muscle EtnGpl were 18:0, EPA, and DHA, while PlsEtn-18:0/20:5, PlsEtn-18:0/22:6, and phosphatidylethanolamine (PtdEtn)-18:0/22:6 were the main phospholipid species. ${ }^{15}$ The porcine liver contained lower PlsEtn, $7.2 \mathrm{~mol}$ $\%$ EtnGpl compared to ascidian muscle. The prominent acyl carbon chains in porcine liver EtnGpl were 18:0, 18:2n-6, and ARA, while PtdEtn-18:0/20:4 was the main phospholipid species. Ascidian muscle EtnGpl had higher ratios of $n-3 / n-6$ and (EPA + DHA)/ARA compared to porcine liver EtnGpl.

2.2. Extrinsic EtnGpl Enhances Cell Viability of Differentiated Caco-2 Cells under Inflammatory Stress. Figure 1 shows that differentiated Caco- 2 cells stimulated with

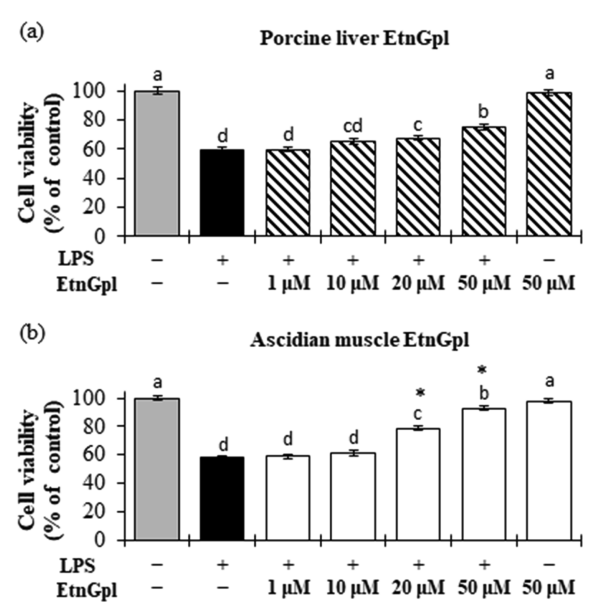

Figure 1. Cell viability during LPS induced inflammatory stress in differentiated Caco- 2 cells. Differentiated Caco- 2 cells were cultured for $48 \mathrm{~h}$ with $1-50 \mu \mathrm{M}$ (a) porcine liver EtnGpl and (b) ascidian muscle EtnGpl containing LPS $(50 \mu \mathrm{g} / \mathrm{mL})$. Cell viability was estimated by counting viable cells under a light microscope in two independent experiments. Values represent means \pm SEM, $n=8$. Different letters indicate significant differences at $P<0.05$, determined by ANOVA (Tukey's test). Asterisks indicate significantly higher cell viability in ascidian muscle EtnGpl-treated cells than porcine liver EtnGpl-treated cells ( $t$ test, $* P<0.05$ ). lipopolysaccharide (LPS; control) had significantly reduced cell viability compared to the blank (untreated cells) $(P<$ $0.05)$. Treatment of differentiated Caco-2 cells with increasing concentrations of EtnGpl under LPS-induced inflammatory stress increased the cell viability in a dose-dependent manner (Figure 1). Cells treated with ascidian muscle and porcine liver EtnGpl at 20 and $50 \mu \mathrm{M}$ showed significantly higher cell viability than those treated with LPS $(P<0.05)$. Moreover, ascidian muscle EtnGpl led to significantly higher cell viability compared to porcine liver EtnGpl at 20 and $50 \mu \mathrm{M}(P<0.05)$.

2.3. Extrinsic EtnGpl Inhibits Apoptosis by Modulating Apoptosis Related Proteins in Inflammatory Stressed Differentiated Caco-2 Cells. Induction of apoptosis by LPS and the subsequent inhibition by extrinsic EtnGpl was evaluated by identifying apoptotic cells in TUNEL assay and cells that showed characteristic morphological changes as determined by DAPI staining (Figure $2 a-d$ ).

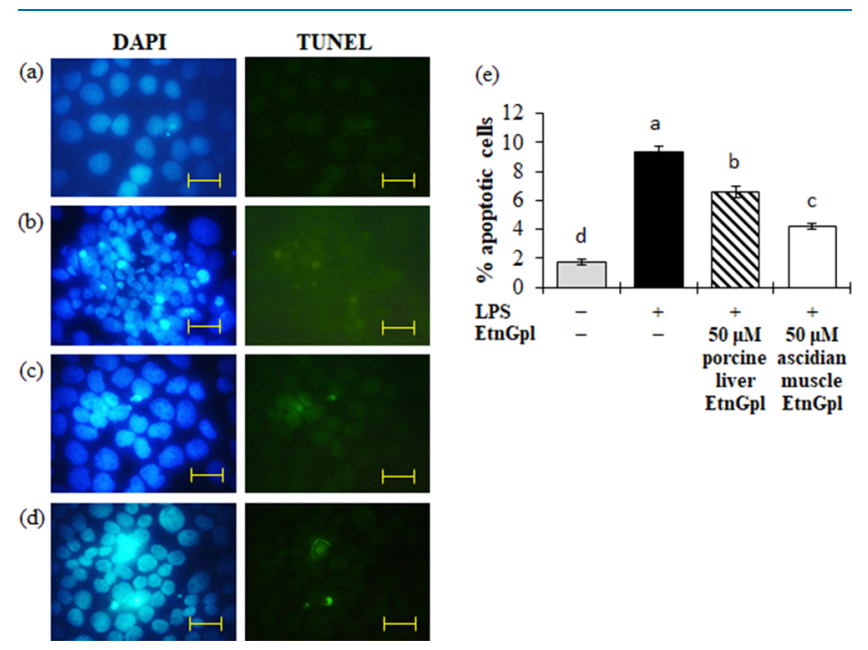

Figure 2. Apoptotic cells during LPS induced inflammatory stress in differentiated Caco-2 cells. Differentiated Caco- 2 cells were cultured for $48 \mathrm{~h}$ with $50 \mu \mathrm{M}$ porcine liver or ascidian muscle EtnGpl containing LPS $(50 \mu \mathrm{g} / \mathrm{mL})$ and then stained with TdT-mediated dUTP nick end labeling (TUNEL) immunofluorescence followed by staining with 4',6-diamidino-2-phenylindole (DAPI). Representative images (objective, 100× ). (a) Blank. (b) LPS (control). (c) LPS + 50 $\mu \mathrm{M}$ porcine liver EtnGpl. (d) LPS $+50 \mu \mathrm{M}$ ascidian muscle EtnGpl. Scale bar indicates $20 \mu \mathrm{m}$. (e) Induction of apoptosis in differentiated Caco- 2 cells by LPS. Values represent means \pm SEM, $n=3$. Different letters indicate significant differences at $P<0.05$, determined by ANOVA (Tukey's test).

Differentiated Caco-2 cells treated with LPS showed blebbing of the membrane and aggregated and fragmented nuclei (Figure 2b). Differentiated Caco-2 cells exposed to LPS showed a significantly greater number of apoptotic-like cells when compared to the blank $(P<0.05)$ (Figure $2 \mathrm{e})$. When differentiated Caco-2 cells were exposed to LPS and EtnGpl, the degree of apoptotic cell death was significantly reduced compared with the LPS treatment, with ascidian muscle EtnGpl showing greater suppression of apoptosis compared to porcine liver EtnGpl $(P<0.05)$.

To gain further insight into the inhibition of apoptosis by EtnGpl during LPS-induced inflammatory stress, we evaluated the relative levels of apoptosis-related proteins (Figure 3). LPS-treated differentiated Caco-2 cells showed significantly higher levels of proapoptotic Bad and cytochrome $c$ compared to blank cells $(P<0.05)$. Furthermore, LPS treatment 

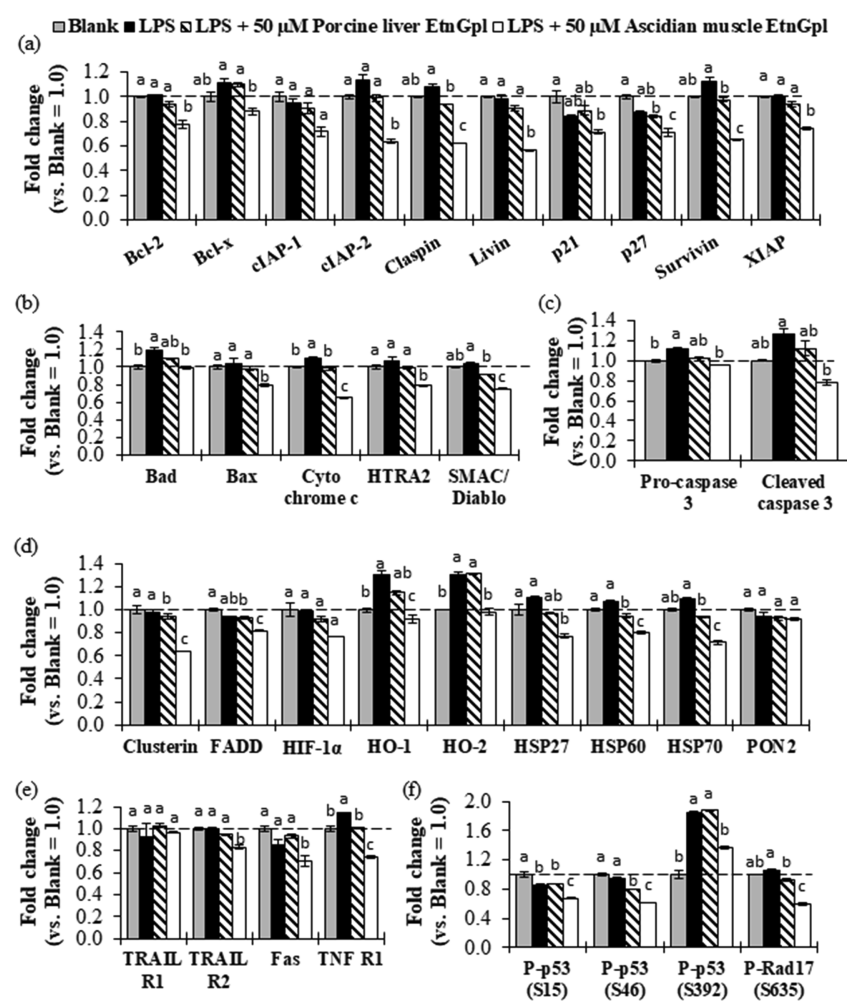

Figure 3. Relative levels of apoptosis-related proteins (fold change vs blank) during LPS induced inflammatory stress in differentiated Caco2 cells. (a) Antiapoptotic proteins. (b) Proapoptotic proteins. (c) Cysteine proteases. (d) Other apoptosis-related proteins. (e) Death receptors. (f) Antibody proteins. The dashed line indicates the blank group $=1.0$. Different letters indicate significant differences at $P<$ 0.05, determined by ANOVA (Tukey's test). For abbreviations, refer to Section 4.5.

significantly upregulated apoptosis executioner pro-caspase 3, TNF R1 death receptor, and P-p53 (S392) antibody protein $(P<0.05)$, and showed a tendency to upregulate SMAC/ Diablo and cleaved caspase 3 compared to the blank cells. Notably, co-treatment with LPS and ascidian muscle EtnGpl significantly downregulated the relative levels of Bad, cytochrome $c$, and SMAC/Diablo proapoptotic proteins, claspin and survivin antiapoptotic proteins, TNF R1 death receptor, P-p53 (S392) antibody protein, and pro-caspase 3, and cleaved caspase 3 apoptosis executioner proteins compared to the LPS-treated cells $(P<0.05)$. On the other hand, although LPS and porcine liver EtnGpl co-treatment downregulated the aforementioned apoptosis-related proteins, the effect was less remarkable compared to that observed with LPS and ascidian muscle EtnGpl co-treatment.

2.4. Extrinsic EtnGpl Ameliorates Inflammation in Differentiated Caco-2 Cells by Lowering Cytokine Levels. A cytokine array assay was conducted to determine the effect of extrinsic EtnGpl on human intestinal tract cells under inflammatory stress (Figure 4). Treatment of differentiated Caco-2 cells with LPS led to higher relative levels of eight pro-inflammatory cytokines, anti-inflammatory cytokines, MCP-1, MIP- $1 \beta$, and SDF-1 chemokines and CD-40 ligand and G-CSF cytokines, while C5/C5a, GRO $\alpha$, IP-10, and IL-8 chemokines were decreased compared to the blank cells (Figure $4 a-d$ ). Co-treatment with LPS and ascidian muscle EtnGpl downregulated 11 pro-inflammatory cytokines, four anti-inflammatory cytokines, four chemokines, and SDF-1 $\square$ Blank $\backsim$ LPS $\triangle \mathrm{LPS}+50 \mu \mathrm{M}$ Porcine liver Etn Gpl $\square \mathrm{LPS}+50 \mu \mathrm{M}$ Ascidian muscle EtnGpl
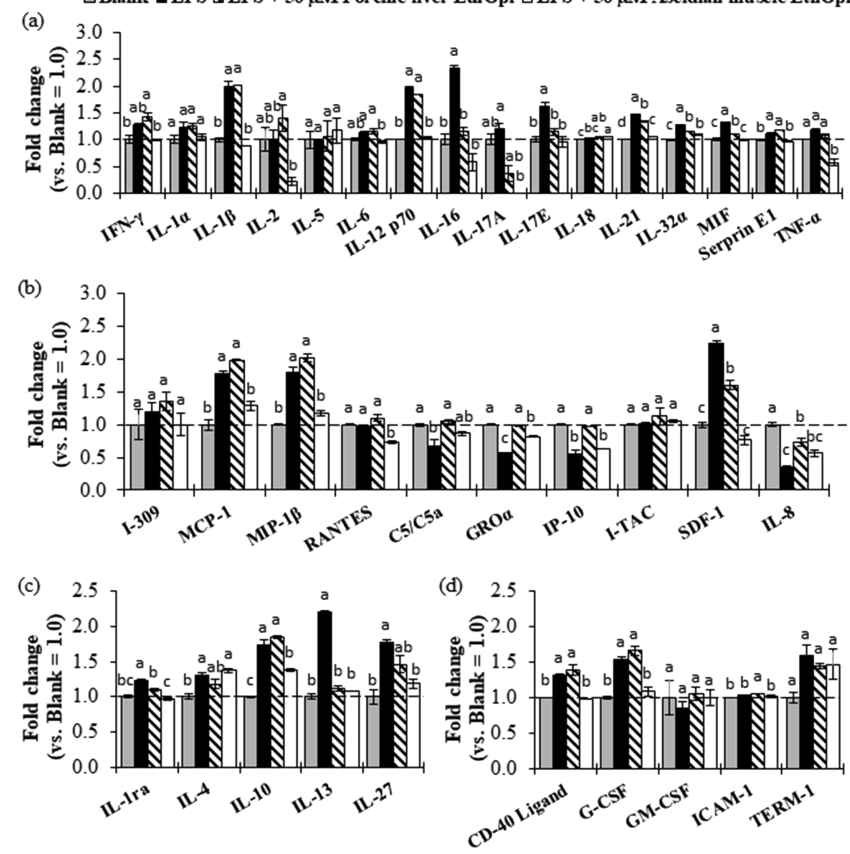

Figure 4. Relative levels of cytokines (fold change vs blank group = 1.0) during LPS induced inflammatory stress in differentiated Caco-2 cells. (a) Pro-inflammatory cytokines. (b) Chemokines. (c) Antiinflammatory cytokines. (d) Other cytokines. The dashed line indicates the blank group $=1.0$. Different letters indicate significant differences at $P<0.05$, determined by ANOVA (Tukey's test). For abbreviations, refer to Section 4.6 .

chemokine, while co-treatment with LPS and porcine liver EtnGpl downregulated five pro-inflammatory cytokines, two anti-inflammatory cytokines, and SDF-1 chemokine (Figure $4 a-d)$. However, four chemokines were upregulated in porcine liver EtnGpl-treated cells compared to LPS-treated cells.

2.5. Uptake of Extrinsic EtnGpl by Differentiated Caco-2 Cells. The lipid composition of the cell lysate was determined to characterize the phospholipid species, acyl, and alkenyl carbon chains of EtnGpl taken up by differentiated Caco-2 cells over time (Figure 5 and Figure S1). Acyl and alkenyl carbon chains of EtnGpl taken up by differentiated Caco-2 cells under LPS treatment were analyzed over time (Figure S2). As shown in Figure 5, we observed that the levels of PlsEtn-18:0/20:4, PlsEtn-18:0/20:5, and PlsEtn-18:0/22:6 from ascidian muscle EtnGpl moved up from 0 to $24 \mathrm{~h}$, with a peak at $16 \mathrm{~h}$ except for PlsEtn-18:0/20:4. The levels of PtdEtn18:0/20:4 from porcine liver EtnGpl showed a steady increase from 0 to $24 \mathrm{~h}$. The levels of choline plasmalogen (PlsCho) and the other PtdEtn and phosphatidylcholine (PtdCho) species from ascidian muscle and porcine liver EtnGpl remained unchanged during the exposure time, except for PtdCho-18:0/20:4 from ascidian muscle EtnGpl, which showed a decrease. Meanwhile, EtnGpl hydrolysis products, lysoPlsEtn-18:0 from ascidian muscle EtnGpl increased during the $24 \mathrm{~h}$ uptake time with a peak at $16 \mathrm{~h}$, while lysoPtdEtn16:0 and 18:0 from ascidian muscle and porcine liver EtnGpl increased in a similar manner with a peak at $16 \mathrm{~h}$.

In addition, we determined the change in the acyl and alkenyl carbon chains over time. As shown in Figure S1, we observed that 18:0ol and EPA levels from ascidian muscle EtnGpl increased from 0 to $24 \mathrm{~h}$ and reached a peak at $16 \mathrm{~h}$. DHA from ascidian muscle EtnGpl decreased in the first $4 \mathrm{~h}$ 

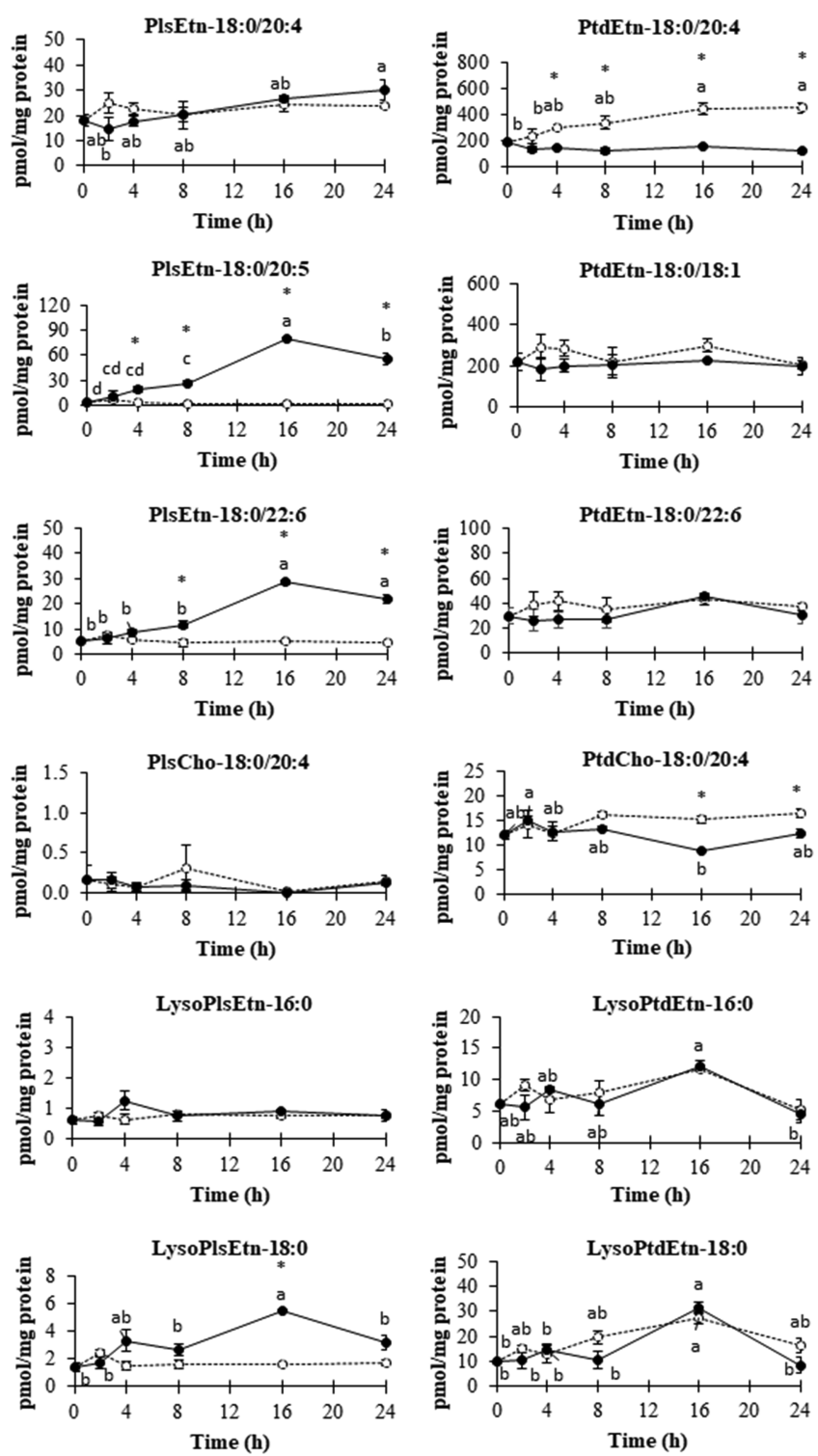

- - $-50 \mu \mathrm{M}$ Porince liver EtnGpl $\longrightarrow 50 \mu \mathrm{M}$ Ascidian muscle EtnGpl

Figure 5. Time-dependent changes in the levels of various phospholipids in differentiated Caco-2 cells. Values represent means \pm SEM, $n=3$. Different letters indicate significant differences at $P<$ 0.05 among cells treated with the same EtnGpl determined by ANOVA (Tukey's test). Asterisks indicate significant differences between cells treated with different EtnGpl. ( $t$ test, $* P<0.05)$.

followed by an increase up to $24 \mathrm{~h}$, with a peak at $16 \mathrm{~h}$. Alkenyl and acyl carbon chains remained unchanged during porcine liver EtnGpl uptake. On the other hand, the changes in acyl and alkenyl carbon chains under LPS treatment over time are shown in Figure S2. For 18:0ol, EPA, and DHA from ascidian muscle EtnGpl, a sharp increase in the first $2 \mathrm{~h}$ followed by a steady increase up to $24 \mathrm{~h}$ was observed. For porcine liver EtnGpl, we observed a steady increase in ARA over time while the other acyl and alkenyl carbon chains remained unchanged.

2.6. Extrinsic EtnGpl Suppresses TNF- $\alpha$ in Differentiated Caco-2 Cells under Inflammatory Stress. TNF$\alpha$ is a major mediator of inflammation and apoptosis in cells. To examine if EtnGpl suppressed inflammation and apoptosis, TNF- $\alpha$ was quantified in differentiated Caco- 2 cells with LPS and EtnGpl-treatment using an ELISA kit (Figure 6).

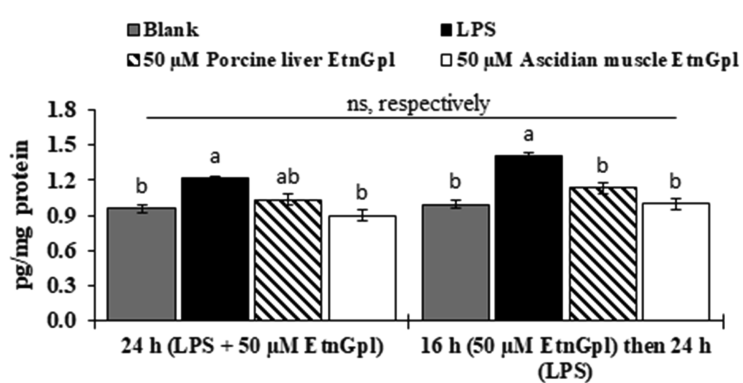

Figure 6. TNF- $\alpha$ levels during LPS induced inflammatory stress in differentiated Caco-2 cells. (a) Differentiated Caco-2 cells were cultured for $24 \mathrm{~h}$ with LPS $+50 \mu \mathrm{M} \mathrm{EtnGpl,} \mathrm{and} \mathrm{in} \mathrm{another}$ experiment, differentiated Caco-2 cells were treated with $50 \mu \mathrm{M}$ EtnGpl for $16 \mathrm{~h}$ (pre-treatment) followed by $50 \mu \mathrm{g} / \mathrm{mL}$ of LPS for 24 $\mathrm{h}$ (post-treatment after EtnGpl was taken up). Values represent means \pm SEM, $n=3$. Different letters indicate significant differences at $P<$ 0.05 , determined by ANOVA (Tukey's test). ns, not significant $(t$ test, $* P<0.05)$.

Differentiated Caco-2 cells treated with LPS for $24 \mathrm{~h}$ demonstrated upregulation of TNF- $\alpha$ compared to the blank cells. Cells cultured for $24 \mathrm{~h}$ in the presence of LPS and EtnGpl exhibited lower levels of TNF- $\alpha$ compared with LPS-treated cells. Moreover, cells treated with LPS and ascidian muscle EtnGpl showed significantly reduced TNF- $\alpha$ levels (almost similar to those in blank cells) compared to LPS-treated cells $(P<0.05)$. In addition, TNF- $\alpha$ levels were lower in LPS and ascidian muscle EtnGpl-treated cells than in LPS and porcine liver EtnGpl-treated cells. On the other hand, there were no significant differences $(P<0.05)$ in TNF- $\alpha$ levels between differentiated Caco- 2 cells co-treated with EtnGpl and LPS in the same medium and those treated with EtnGpl first followed by LPS. The treatment of the cells with EtnGpl for $16 \mathrm{~h}$ was based on our uptake experiment, which showed that uptake of EtnGpl by differentiated Caco-2 cells reached a peak at $16 \mathrm{~h}$. This suggests that the uptake of EtnGpl by the cells led to the observed functionality during LPS-induced inflammatory stress.

\section{DISCUSSION}

In the present study, we demonstrate that an increase in intracellular PlsEtn levels is associated with anti-inflammatory and antiapoptotic effects in human intestinal tract cells. We employed LPS for the induction of inflammatory stress in vitro, which has been extensively used in studies on proinflammatory and proapoptotic responses in differentiated Caco-2 cells. ${ }^{16,41}$ Moreover, in vitro epithelial cell studies have been shown to play a critical role in understanding specific aspects related to IBD. ${ }^{17}$ Here, using an in vitro model, we observed that in comparison to extrinsic EtnGpl with low PlsEtn content, the one having high PlsEtn content and distinct PUFA at the $s n-2$ position showed superior inhibition of inflammation and apoptosis in human intestinal tract cells under LPS-induced inflammatory stress via downregulation of inflammatory cytokines and modulation of apoptosis-related proteins. Extrinsic EtnGpl from the ascidian muscle was found to maintain higher intracellular levels of PlsEtn species compared to that from the porcine liver. These results suggest that the uptake of PlsEtn by colon epithelial cells provides protection against induced inflammatory stress by suppressing apoptosis. This evidence shows a clear relationship between 
increased PlsEtn levels in colon epithelial cells and attenuation of tissue injury during induced stress.

Chronic colon inflammation has been closely associated with the development of IBD and colon cancer., 18 Chronic inflammation induced by stress stimuli has been linked to the deregulation of apoptosis, which disrupts normal cellular homeostasis. ${ }^{19}$ In neuronal cells under serum starvation, EtnGpl containing high PlsEtn levels suppresses the activities of caspases 3,8 , and 9, which are involved in the mitochondrial and death receptor pathways. ${ }^{4}$ In our study, LPS treatment upregulated proapoptotic Bad and cytochrome $c$, while cotreatment with LPS and EtnGpl downregulated their relative levels (ascidian muscle > porcine liver EtnGpl) (Figure 3b). Moreover, the uptake of ascidian muscle EtnGpl resulted in higher levels of PlsEtn species, EPA, and DHA compared to porcine liver EtnGpl. Based on these findings, we suggest that the uptake of PlsEtn might have limited the release of proapoptotic cytochrome $c$ into the cytosol. The release of cytochrome $c$ activates initiator caspase 9, which in turn, activates the apoptosis executioner caspase $3 .{ }^{10}$ Our results further confirmed the possibility of a limited release of cytochrome $c$ due to the downregulation of apoptosis executioner pro-caspase 3 and cleaved caspase 3 by LPS and EtnGpl co-treatment (ascidian muscle > porcine liver EtnGpl) compared to LPS treatment (Figure 3c). Moreover, phosphorylation of p53 protein is known to trigger the induction of apoptosis via activation of proapoptotic related protein, leading to the release of cytochrome $c .{ }^{20}$ We observed that LPS treatment upregulated P-p53 (S392) and not P-p53 (S15 and S46); however, all were downregulated by ascidian muscle EtnGpl and LPS co-treatment. Upon DNA damage, phosphorylation of p53 at S394 is within its COOH-terminal region that enhances p53 DNA binding activity while that of p53 at $\mathrm{S} 15$ and $\mathrm{S} 46$ is within its $\mathrm{NH}_{2}$-termimal region that promotes dissociation of MDM2, which might possibly explain the observed differences. ${ }^{21}$ Taken altogether, this explains the observed effect on cell viability and suppression of apoptotic cells by PlsEtn (ascidian muscle EtnGpl > porcine liver EtnGpl $>$ LPS).

TNF- $\alpha$ is known to control inflammatory cell populations and mediate many other aspects of the inflammatory process, contributing to the disruption of the intestinal epithelial barrier. $^{18}$ It has been shown that i.p. administration of plasmalogens (Pls) reduces the expression of TNF- $\alpha$ in the hippocampus of LPS-treated mice. ${ }^{22}$ In our in vitro study, we found that extrinsic EtnGpl suppressed the LPS-induced high levels of TNF- $\alpha$. Furthermore, TNF- $\alpha$ is implicated in the induction of apoptosis involving the death receptor TNF-R1 via the extrinsic apoptosis pathway. ${ }^{10}$ In the present study, we observed that extrinsic EtnGpl lowered the relative levels of TNF-R1 induced by LPS (ascidian muscle > porcine liver EtnGpl) (Figure 3c). It is possible that EtnGpl might have suppressed caspase-dependent apoptosis via the extrinsic pathway. Cytokines are important in the pathogenesis of colon inflammation, and their manipulation can reduce disease severity and maintain remission. Cellular secretion of proinflammatory cytokines mediates many responses, including apoptosis during chronic colon inflammation. ${ }^{23}$ We showed here that an increase in pro-inflammatory cytokines by LPS treatment was downregulated by extrinsic EtnGpl (ascidian muscle > porcine liver EtnGpl), which possibly generated a favorable microenvironment for the survival of the cells.
PlsEtn has been shown to form more condensed and thicker membranes, possibly due to the vinyl ether linkage at the $s n-1$ position. ${ }^{24}$ Furthermore, EPA-PlsEtn exerts a better effect in lowering amyloid- $\beta$ levels in CHO-APP/PS1 cells in vitro, which is partly attributed to the vinyl ether linkage. ${ }^{25}$ In the present study, the observed increase in PlsEtn levels in differentiated Caco-2 cells (ascidian muscle > porcine liver EtnGpl) might have influenced the intracellular microenvironment differently. Moreover, chemically induced inflammatory stress is associated with reactive oxygen species (ROS), and ROS play a central role in the regulation of main pathways in apoptosis. $^{26}$ However, the vinyl ether linkage at the $s n-1$ position makes Pls more susceptible to oxidative stress. ${ }^{27}$ Indeed, it has been demonstrated that one vinyl ether double bond in Pls can scavenge two peroxy radicals. ${ }^{28,29}$ This might partly explain the superior antiapoptotic effect of the ascidian muscle EtnGpl over the porcine liver EtnGpl observed in our study (Figures 1 and 2). Therefore, PlsEtn might have acted as an antioxidant and protected cells from LPS-induced inflammatory stress.

Ascidian muscle EtnGpl containing EPA and DHA had a superior antiapoptotic effect compared to porcine liver EtnGpl containing ARA. Extrinsic ascidian muscle EtnGpl yielded higher levels of intracellular PlsEtn containing DHA and EPA compared to porcine liver EtnGpl (Figure 5 and Figures S1 and S2). PlsEtn-selective phospholipase $A_{2}$ is activated by inflammation, and quarried EPA and DHA give rise to eicosanoids and docosanoids, which serve as inflammationresolving mediators. ${ }^{30,31}$ This changes the pattern of cellular inflammatory mediators and decreases chemically induced colonic damage and inflammation. ${ }^{32}$ In this study, ascidian muscle EtnGpl, which led to increased PlsEtn containing EPA and DHA, showed a higher suppression of apoptotic responses in the cells. Furthermore, since ARA is metabolized to form eicosanoids that serve as inflammatory mediators, the observed increase in PtdEtn-containing ARA with porcine liver EtnGpl could possibly explain the relatively similar levels of a few proinflammatory cytokines between porcine liver EtnGpl-treated cells and LPS-treated cells.

During intestinal absorption of phospholipids, fatty acids are released as a result of hydrolysis at the $s n-2$, which yields lysophospholipids. ${ }^{33}$ Lysophospholipids have been identified as biologically active lipid mediators. ${ }^{34}$ For instance, lysoPtdCho bearing DHA have been shown to exhibit anti-inflammatory effects in vivo and in vitro in mouse and macrophage models, respectively. ${ }^{35}$ In the present study, we observed an increase in lysoPlsEtn-18:0 from ascidian muscle EtnGpl and an increase in lysoPtdEtn-18:0 from both EtnGpl treatments (Figure 5). This possibly suggests their involvement in suppressing colon inflammation and apoptosis during LPS-induced stress. We have recently demonstrated that lysoEtnGpl improves the absorption kinetics of PlsEtn in vivo in the plasma of mice through re-esterification. ${ }^{36}$ Therefore, it would be interesting to elucidate the protective role of lysoPlsEtn vis-à-vis PlsEtn during colon inflammation and carcinogenesis in vivo and in vitro. Thus, further detailed studies to clarify the food functionality of lysoPlsEtn in the colon should be conducted.

In conclusion, our findings provide evidence that the elevation of PlsEtn in colon epithelial cells might contribute to the alleviation of stress-induced inflammation and apoptosis responses. The resulting intracellular antiapoptotic effect was achieved via suppression of pro-inflammatory cytokines and inhibition of proapoptotic proteins. Our present research 
findings suggest that the intake of PlsEtn with abundant vinyl ether linkages and n-3 PUFA efficiently inhibits the downstream inflammatory and apoptotic signaling cascade in the colon. Therefore, PlsEtn derived from food sources with high vinyl ether linkages and n-3 PUFA is potentially beneficial in averting the initiation of IBD and colon cancer, which are strongly associated with chronic colon inflammation.

\section{MATERIALS AND METHODS}

4.1. Materials. Materials used in the cell culture experiment were purchased as follows: human colon carcinoma cell line Caco-2 from Riken Gene Bank (Tsukuba, Japan); Dulbecco's modified Eagle's medium (DMEM), trypsinEDTA $(\times 10)$, and protease inhibitor cocktail for use with mammalian cell and tissue extracts from Sigma-Aldrich, Inc. (Missouri, MO, USA); lysis buffer 17 from R\&D Systems (Minneapolis, MN, USA); phosphate-buffered saline (PBS) from Nissui (Tokyo, Japan); fetal bovine serum (FBS) from Biowest (Nuaille, France); penicillin-streptomycin-amphotericin B $(\times 100)$, MEM non-essential amino acids $(\times 100)$, LPS, and bovine serum albumin (BSA) fatty acid free from Fujifilm Wako Pure Chemical Corp. (Gunma, Osaka, Japan); and phospholipid species from Avanti Polar Lipids (Alabaster, AL, USA). PlsEtn-18:0/20:5 was purified according to our previously reported method. ${ }^{37}$

4.2. Ascidian Muscle and Porcine Liver EtnGpl Preparation. Purified ascidian muscle and porcine liver EtnGpl were prepared as previously described in our study. ${ }^{37}$ Purified EtnGpl was dissolved in ethanol to a stock solution of $10 \mathrm{mM}$ and diluted to $0.5 \%$ ethanol by adding to DMEM containing $0.1 \% \mathrm{BSA}$ at the time of use.

4.3. Cell Culture. The human colon carcinoma cell line Caco- 2 was cultured in DMEM supplemented with $10 \%$ heatinactivated FBS (v/v), 1\% penicillin-streptomycin-amphoteric $\mathrm{B}(\mathrm{v} / \mathrm{v})$, and $1 \%$ non-essential amino acids (v/v) in an incubator at $37{ }^{\circ} \mathrm{C}$ and $5 \% \mathrm{CO}_{2}$ under humid conditions. Cells were continuously passaged at $1.5 \times 10^{6}$ cells $/ \mathrm{mL}$ in $100 \mathrm{~mm}$ dishes every 3-4 days. Differentiated Caco-2 cells were obtained by seeding Caco- 2 cells, and when they reached $>90 \%$ confluence, they were designated as day 0 and incubated for 21 days to differentiate. In this study, differentiated Caco-2 cell medium was changed to DMEM with $0.1 \%$ BSA containing $50 \mu \mathrm{g} / \mathrm{mL}$ LPS (control), LPS, and ascidian muscle or porcine liver EtnGpl ( 1 to $50 \mu \mathrm{M}$ ), and a blank treatment. The dose levels of EtnGpl used in this study were based on our previous study in which 5 to $50 \mu \mathrm{M}$ of EtnGpl increased the viability of serum-starved Neuro-2A cells. ${ }^{38}$

4.4. Cell Viability. Caco- 2 cells at a concentration of $2.0 \times$ $10^{5}$ were seeded into 24 -well plates (Nunc, Rochester, NY) containing $1 \mathrm{~mL}$ of culture medium. The cells were incubated for the indicated times and conditions. Differentiated Caco-2 cells were then treated with LPS $(50 \mu \mathrm{g} / \mathrm{mL})$ and EtnGpl in a dose-dependent manner $(1,10,20$, and $50 \mu \mathrm{M})$ for $48 \mathrm{~h}$ followed by rinsing with pre-warmed PBS, trypsinized, and counted using a counting chamber (EM-Techcolor; Hirschmann, Eberstadt, Germany).

4.5. Apoptosis Detection. Caco-2 cells at a concentration of $1.0 \times 10^{5}$ were seeded in eight-well Lab-Tek chamber slides (Thermo Scientific, Waltham, MA) containing $0.5 \mathrm{~mL}$ of culture medium. Differentiated Caco-2 cells were then treated with $50 \mu \mathrm{M}$ EtnGpl and LPS for $24 \mathrm{~h}$. Apoptotic cells were visualized and determined by double staining method using the TACS 2 TdT-Flour in situ apoptosis detection kit (Trevigen) as per manufacturer's instruction followed by DAPI staining under a fluorescence microscope (BX-51; Olympus, Tokyo, Japan). The percentage of apoptotic cells was determined by dividing the number of apoptotic cells by the total number of cells and multiplying by $100 .^{39-41}$

To confirm the results of morphological analysis, the relative levels of apoptosis-related proteins were examined using the Human Apoptosis Array Kit (R\&D Systems, Minneapolis, $\mathrm{MN})$. Briefly, Caco- 2 cells were seeded in $100 \mathrm{~mm}$ dishes $(1.5$ $\times 10^{6}$ cells) and incubated as indicated. Differentiated Caco-2 cells were treated with $50 \mu \mathrm{M}$ EtnGpl and LPS $(50 \mu \mathrm{g} / \mathrm{mL})$ for $24 \mathrm{~h}$ and then rinsed with cold PBS followed by lysis with cold lysis buffer 17 containing $10 \mu \mathrm{L} / \mathrm{mL}$ protease inhibitor cocktail. Membranes coated with 35 different anti-apoptosisrelated antibodies were exposed to the cell lysate, and apoptosis-related proteins were detected according to the manufacturer's protocol. Data of captured antibody pixel densities from an image of the developed X-ray film were acquired using ImageJ software. The detected apoptosis-related proteins were denoted as follows: $\mathrm{Bcl}-\mathrm{xL} / \mathrm{Bcl}-2$ associated death promoter (Bad), Bcl-2-associated X protein (Bax), B-cell lymphoma 2 ( $\mathrm{Bcl}-2), \mathrm{Bcl} /$ leukemia $\mathrm{x}(\mathrm{Bcl}-\mathrm{x})$, pro-caspase 3, cleaved caspase 3 , catalase, cytosolic inhibitors of apoptosis-1 (cIAP-1), cytosolic inhibitors of apoptosis-2 (cIAP-2), claspin, clusterin, cytochrome $c$, TNF receptor 1 (TNF R1), TNFrelated apoptosis-inducing ligand receptor 1 (TRAIL R1), TRAIL R2, Fas associated protein with death domain (FADD), fibroblast-associated (Fas), hypoxia-inducible transcription factor (HIF)-1 $\alpha$, heme oxygenase (HO)-1, HO-2, heat shock protein (HSP)27, HSP60, HSP70, high temperature requirement protein A2 (HTRA2/Omi), livin, paraoxonase 2 (PON2), cyclin-dependent kinase 4 inhibitor 1 (p21), cyclin-dependent kinase 4 inhibitor 1 (p27), phosphorylated p53 at serine 15 [phospho-p53 (S15)], phosphop53 (S46), phospho-p53 (S392), phospho-Rad17 (S635), second mitochondria-derived activator of caspase/direct inhibitor of apoptosis-binding protein with low pI (SMAC/ Diablo), surviving, and X-linked inhibitor of apoptosis (XIAP).

4.6. Cytokine Array. Relative cytokine levels were determined using a Human Cytokine Array Kit (R\&D Systems, Minneapolis, MN). The cells were cultured, incubated, and treated as indicated in the analysis of apoptosis-related proteins. Membranes coated with 36 different anti-cytokine antibodies were exposed to the cell lysate, and cytokines were detected according to the manufacturer's protocol. Then, data were acquired as described above. The detected cytokines were denoted as follows: chemokine (C-C motif) ligand 1 (CCL1/I-309), chemokine (C-C motif) ligand 2 (CCL2)/monocyte chemoattractant protein 1 (MCP-1), macrophage inflammatory protein-1 alpha (MIP-1 $\alpha$ :CCL3)/ macrophage inflammatory protein-1 beta (MIP-1 $\beta$ :CCL4), regulated on activation, normal $\mathrm{T}$ cell expressed and secreted (RANTES), CD-40 ligand, complement component 5a (C5/ $\mathrm{C} 5 \mathrm{a})$, growth related oncogene-alpha (GRO- $\alpha$ ), interferon- $\gamma$ induced protein 10 (IP-10), interferon-inducible $\mathrm{T}$ cell alpha chemoattractant (I-TAC), stromal cell-derived factor 1 (SDF1), granulocyte colony stimulating factor (G-CSF), granulocyte macrophage colony-stimulating factor (GM-CSF), intercellular adhesion molecule 1 (ICAM-1), interferon-gamma (IFN- $\gamma$ ), interleukins (IL-1 $\alpha$, IL- $1 \beta$, IL-1ra, IL-2, IL-4, IL-5, IL-6, IL-8, IL-10, IL-12p70, IL-13, IL-16, IL-17A, IL-17E, IL-18, IL-21, IL-27, and IL-32a), macrophage migration inhibitory factor (MIF), plasminogen activator inhibitor-1 (PAI-1:Serpin E1), 
TNF- $\alpha$, and triggering receptor expressed on myeloid cells 1 (TREM-1).

4.7. TNF- $\alpha$ Assay. TNF- $\alpha$ levels were quantified using a Human TNF- $\alpha$ ELISA kit (FUJIFILM Wako Shibayagi Corp., Gunma, Japan). Briefly, the cells were cultured and incubated as indicated. The differentiated cells were treated for $24 \mathrm{~h}$, and the cell lysates were collected as described. Additionally, differentiated Caco- 2 cells were treated with $50 \mu \mathrm{M}$ EtnGpl for $16 \mathrm{~h}$ followed by LPS $(50 \mu \mathrm{g} / \mathrm{mL})$ treatment for $24 \mathrm{~h}$ to confirm whether LPS inhibited the functionality and/or uptake of EtnGpl by differentiated Caco- 2 cells. The cell lysates (20 $\mu \mathrm{L}$ ) were added to 96-well microplates with the standards, and the assay was performed according to the manufacturer's protocol. Absorbance at 450 and $620 \mathrm{~nm}$ was read using a 96well microplate read (ThermoScietific Multiskan FC version 2.5, Finland). Protein contents of samples were measured using a DC protein assay kit (Bio-rad, CA, USA).

4.8. Lipid Extraction and Assay. To determine the uptake of extrinsic EtnGpl by differentiated Caco-2 cells, fatty acid methyl esters (FAME), dimethyl acetals (DMA), and phospholipid species in cell lysates were analyzed over a period of $24 \mathrm{~h}$. Briefly, differentiated Caco- 2 cells were treated with 50 $\mu \mathrm{M}$ EtnGpl for $0,2,4,8,16$, and $24 \mathrm{~h}$ with $0.1 \%$ BSA instead of $10 \%$ FBS. At each time interval, the cell surface binding lipids were removed by washing the cells twice with $20 \mathrm{mmol} /$ $\mathrm{L}$ Tris $-\mathrm{HCl}, 2 \mathrm{~mol} / \mathrm{L} \mathrm{NaCl}, \mathrm{pH} 4.0 .^{42}$ The cells were scraped off the plate, ultrasonicated for $10 \mathrm{~s}$ on ice, and stored at -80 ${ }^{\circ} \mathrm{C}$ until use. Total lipids were extracted according to the Folch method. ${ }^{43}$ To obtain the phospholipid fraction, $1 \mathrm{~mL}$ of the extracted total lipids was dried by $\mathrm{N}_{2}$ flux and dissolved in 200 $\mu \mathrm{L}$ of chloroform-isopropanol (2:1), and then loaded onto a silica Sep-Pak cartridge (Waters, Tokyo, Japan) in which very fine silica particles had been removed by passing $1.5 \mathrm{~mL}$ of methanol and equilibrated with $1.5 \mathrm{~mL}$ of chloroformisopropanol (2:1). The loaded sample was eluted with $1.5 \mathrm{~mL}$ of chloroform-isopropanol $(2: 1)$ to remove nonpolar compounds followed by elution with $1.5 \mathrm{~mL}$ of methanol, and the eluted methanol solution was collected as the phospholipid fraction. FAME and DMA prepared from the lipid extracts were analyzed by gas chromatography. ${ }^{44}$ Phospholipid species were analyzed by LC-MS/MS in multiple reaction monitoring (MRM) mode. ${ }^{45}$

4.9. Statistical Analysis. The results are represented as mean \pm SEM. All data were subjected to analysis of variance using SPSS software (version 17.0; SPSS Inc., Chicago, IL, USA). Differences between the means were tested using oneway ANOVA followed by Tukey's post hoc test. $P<0.05$ was considered statistically significant.

\section{ASSOCIATED CONTENT}

\section{SI Supporting Information}

The Supporting Information is available free of charge at https://pubs.acs.org/doi/10.1021/acsomega.0c05545.

Time-dependent changes of phospholipid carbon chain composition (alkenyl and acyl) in differentiated Caco-2 cells treated with $50 \mu \mathrm{M}$ porcine liver and ascidian muscle EtnGpl with and without LPS (PDF)

\section{AUTHOR INFORMATION}

\section{Corresponding Author}

Shinji Yamashita - Department of Life and Food Sciences, Obihiro University of Agriculture and Veterinary Medicine,
Obihiro 080-8555, Japan; 이이.org/0000-0001-74435670; Phone: +81-155-49-5446; Email: syamashita@ obihiro.ac.jp; Fax: +81-155-49-5593

\section{Authors}

Ephantus Nguma - Department of Life and Food Sciences, Obihiro University of Agriculture and Veterinary Medicine, Obihiro 080-8555, Japan

Kei Kumagai - Food and Biodynamic Chemistry Laboratory, Graduate School of Agricultural Science, Tohoku University, Sendai 980-8572, Japan

Yurika Otoki - Food and Biodynamic Chemistry Laboratory, Graduate School of Agricultural Science, Tohoku University, Sendai 980-8572, Japan

Ayaka Yamamoto - Yaizu Suisankagaku Industry Co., Ltd., Shizuoka 425-8570, Japan

Takahiro Eitsuka - Food and Biodynamic Chemistry Laboratory, Graduate School of Agricultural Science, Tohoku University, Sendai 980-8572, Japan

Kiyotaka Nakagawa - Food and Biodynamic Chemistry Laboratory, Graduate School of Agricultural Science, Tohoku University, Sendai 980-8572, Japan; 이이.org/00000003-3026-7358

Teruo Miyazawa - Food and Biotechnology Platform Promoting Project, New Industry Creation Hatchery Center (NICHe), Tohoku University, Sendai 980-8579, Japan

Mikio Kinoshita - Department of Life and Food Sciences, Obihiro University of Agriculture and Veterinary Medicine, Obihiro 080-8555, Japan

Complete contact information is available at:

https://pubs.acs.org/10.1021/acsomega.0c05545

\section{Notes}

The authors declare no competing financial interest.

\section{ACKNOWLEDGMENTS}

A part of this research was supported by a grant from the Project of NARO Bio-oriented Technology Research Advancement Institution (R\&D matching funds on the field for Knowledge Integration and Innovation) and JSPS KAKENHI grant no. JP19K05892.

\section{ABBREVIATIONS}

ARA, arachidonic acid (20:4n-6); DHA, docosahexaenoic acid (22:6n-3); EPA, eicosapentaenoic acid (20:5n-3); EtnGpl, ethanolamine glycerophospholipid; IBD, inflammatory bowel disease; LPS, lipopolysaccharide; LysoPlsCho, lysocholine plasmalogen; LysoPlsEtn, lysoethanolamine plasmalogen; LysoPtdCho, lysophosphatidylcholine; LysoPtdEtn, lysophosphatidylethanolamine; ns, not significant; PlsCho, choline plasmalogen; PlsEtn, ethanolamine plasmalogen; PtdCho, phosphatidylcholine; PtdEtn, phosphatidylethanolamine.

\section{REFERENCES}

(1) Braverman, N. E.; Moser, A. B. Functions of Plasmalogen Lipids in Health and Disease. Biochim. Biophys. Acta, Mol. Basis Dis. 2012, 1822, 1442-1452.

(2) Brites, P.; Waterham, H. R.; Wanders, R. J. A. Functions and Biosynthesis of Plasmalogens in Health and Disease. Biochim. Biophys. Acta 2004, 1636, 219-231.

(3) Yamashita, S.; Abe, A.; Nakagawa, K.; Kinoshita, M.; Miyazawa, T. Separation and Detection of Plasmalogen in Marine Invertebrates 
by High-Performance Liquid Chromatography with Evaporative Light-Scattering Detection. Lipids 2014, 49, 1261-1273.

(4) Yamashita, S.; Kanno, S.; Honjo, A.; Otoki, Y.; Nakagawa, K.; Kinoshita, M.; Miyazawa, T. Analysis of Plasmalogen Species in Foodstuffs. Lipids 2016, 51, 199-210.

(5) Yamashita, S.; Hashimoto, M.; Haque, A. M.; Nakagawa, K.; Kinoshita, M.; Shido, O.; Miyazawa, T. Oral Administration of Ethanolamine Glycerophospholipid Containing a High Level of Plasmalogen Improves Memory Impairment in Amyloid B-infused Rats. Lipids 2017, 52, 575-585.

(6) Ding, L.; Zhang, L.; Shi, H.; Xue, C.; Yanagita, T.; Zhang, T.; Wang, Y. EPA-Enriched Ethanolamine Plasmalogen Alleviates Atherosclerosis via Mediating Bile Acids Metabolism. J. Funct. Foods 2020, 66, 103824.

(7) Mawatari, S.; Ohara, S.; Taniwaki, Y.; Tsuboi, Y.; Maruyama, T.; Fujino, T. Improvement of Blood Plasmalogens and Clinical Symptoms in Parkinson's Disease by Oral Administration of Ether Phospholipids: A Preliminary Report. Parkinson's. Dis. 2020, 2020, 2671070.

(8) Bray, F.; Ferlay, J.; Soerjomataram, I.; Siegel, R.; Torre, L.; Jemal, A. Global Cancer Statistics 2018: GLOBOCAN Estimates of Incidence and Mortality Worldwide for 36 Cancers in 185 Countries. CA Cancer J. Clin. 2018, 68, 394-424.

(9) Bartsch, H.; Nair, J. Chronic Inflammation and Oxidative Stress in the Genesis and Perpetuation of Cancer: Role of Lipid Peroxidation, DNA Damage, and Repair. Langenbeck's Arch. Surg. 2006, 391, 499-510.

(10) Yang, S. Y.; Sales, K. M.; Fuller, B.; Seifalian, A. M.; Winslet, M. C. Apoptosis and Colorectal Cancer: Implications for Therapy. Trends Mol. Med. 2009, 15, 225-233.

(11) Hofmanová, J.; Straková, N.; Vaculová, A. H.; Tylichová, Z.; Safariková, B.; Skender, B.; Kozubík, A. Interaction of Dietary Fatty Acids with Tumour Necrosis Factor Family Cytokines during Colon Inflammation and Cancer. Mediators Inflamm. 2014, 2014, 848632.

(12) Krzystek-korpacka, M.; Diakowska, D.; Kapturkiewicz, B.; Bebenek, M.; Gamian, A. Profiles of Circulating Inflammatory Cytokines in Colorectal Cancer (CRC), High Cancer Risk Conditions, and Health Are Distinct . Possible Implications for CRC Screening and Surveillance. Cancer Lett. 2013, 337, 107-114.

(13) Hossain, Z.; Hosokawa, M.; Takahashi, K. Growth Inhibition and Induction of Apoptosis of Colon Cancer Cell Lines by Applying Marine Phospholipid. Nutr. Cancer 2009, 61, 123-130.

(14) Messias, M. C. F.; Mecatti, G. C.; Priolli, D. G.; de Oliveira Carvalho, P. Plasmalogen Lipids: Functional Mechanism and Their Involvement in Gastrointestinal Cancer. Lipids Health Dis. 2018, 17, 41.

(15) Nguma, E.; Tominaga, Y.; Yamashita, S.; Otoki, Y.; Yamamoto, A.; Nakagawa, K.; Miyazawa, T.; Kinoshita, M. Dietary Ethanolamine Plasmalogen Ameliorates Colon Mucosa Inflammatory Stress and Pre-Cancerous ACF in 1,2-DMH-Induced Colon Carcinogenesis Mice Model: Protective Role of Vinyl Ether Linkage. Lipids, in press, DOI: $10.1002 /$ lipd.12283.

(16) Jutanom, M.; Higaki, C.; Yamashita, S.; Nakagawa, K.; Matsumoto, S.; Kinoshita, M. Effects of Sphingolipid Fractions from Golden Oyster Mushroom (Pleurotus Citrinopileatus) on Apoptosis Induced by Inflammatory Stress in an Intestinal Tract in Vitro Model. J. Oleo Sci. 2020, 69, 1087-1093.

(17) McKay, D. M.; Philpott, D. J.; Perdue, M. H. Review Article: In Vitro Models in Inflammatory Bowel Disease Research - A Critical Review. Aliment. Pharmacol. Ther. 1997, 11, 70-80.

(18) Coussens, L. M.; Werb, Z. Inflammation and Cancer. Nature 2002, 420, 860-867.

(19) Fulda, S.; Gorman, A. M.; Hori, O.; Samali, A. Cellular Stress Responses: Cell Survival and Cell Death. Int. J. Cell Biol. 2010, 2010, 214074.

(20) Yogosawa, S.; Yoshida, K. Tumor Suppressive Role for Kinases Phosphorylating P53 in DNA Damage-Induced Apoptosis. Cancer Sci. 2018, 109, 3376-3382.
(21) Ozaki, T.; Nakagawara, A. Role of P53 in Cell Death and Human Cancers. Cancers (Basel). 2011, 3, 994-1013.

(22) Ifuku, M.; Katafuchi, T.; Mawatari, S.; Noda, M.; Miake, K.; Sugiyama, M.; Fujino, T. Anti-Inflammatory/Anti-Amyloidogenic Effects of Plasmalogens in Lipopolysaccharide-Induced Neuroinflammation in Adult Mice. J. Neuroinflammation 2012, 9, 197.

(23) Sanchez-muñoz, F.; Dominguez-lopez, A.; Yamamoto-furusho, J. K. Role of Cytokines in Inflammatory Bowel Disease. World J. Gastroenterol. 2008, 14, 4280-4288.

(24) Rubio, J. M.; Astudillo, A. M.; Casas, J.; Balboa, M. A.; Balsinde, J. Regulation of Phagocytosis in Macrophages by Membrane Ethanolamine Plasmalogens. Front. Immunol. 2018, 9, 1723.

(25) Che, H.; Zhou, M.; Zhang, T.; Zhang, L.; Ding, L.; Yanagita, T.; Xu, J.; Xue, C.; Wang, Y. EPA Enriched Ethanolamine Plasmalogens Significantly Improve Cognition of Alzheimer's Disease Mouse Model by Suppressing $\beta$-Amyloid Generation. J. Funct. Foods 2018, 41, 9-18.

(26) Redza-dutordoir, M.; Averill-bates, D. A. Activation of Apoptosis Signalling Pathways by Reactive Oxygen Species. BBABiochimica Biophys. Acta, Mol. Cell Res. 2016, 1863, 2977-2992.

(27) Wallner, S.; Schmitz, G. Plasmalogens the Neglected Regulatory and Scavenging Lipid Species. Chem. Phys. Lipids 2011, $164,573-589$.

(28) Hahnel, D.; Beyer, K.; Engelmann, B. Inhibition of Peroxyl Radical-Mediated Lipid Oxidation by Plasmalogen Phospholipid and $\alpha$-Tocopherol. Free Radical Biol. Med. 1999, 27, 1087-1094.

(29) Leßig, J.; Fuchs, B. Plasmalogens in Biological Systems: Their Role in Oxidative Processes in Biological Membranes, Their Contribution to Pathological Processes and Aging and Plasmalogen Analysis. Curr. Med. Chem. 2009, 16, 2021-2041.

(30) Calder, P. C. Marine Omega-3 Fatty Acids and Inflammatory Processes: Effects, Mechanisms and Clinical Relevance. Biochim. Biophys. Acta, Mol. Cell Biol. Lipids 2015, 1851, 469-484.

(31) Farooqui, A. A. Studies on Plasmalogen-Selective Phospholipase A2 in Brain. Mol. Neurobiol. 2010, 41, 267-273.

(32) Empey, L. R.; Jewell, L. D.; Garg, M. L.; Thomson, A. B. R.; Clandinin, M. T.; Fedorak, R. N. Fish Oil-Enriched Diet Is Mucosal Protective against Acetic Acid-Induced Colitis in Rats. Can. J. Physiol. Pharmacol. 1991, 69, 480-487.

(33) Cohn, J. S.; Kamili, A.; Wat, E.; Chung, R. W. S.; Tandy, S. Dietary Phospholipids and Intestinal Cholesterol Absorption. Nutrients 2010, 2, 116-127.

(34) McMullen, C. A.; Natarajan, V.; Smyth, S. S. Lysophospholipids and Their Receptors: New Data and New Insights into Their Function. Biochim. Biophys. Acta - Mol. Cell Biol. Lipids 2020, 1865, 158697.

(35) Hung, N. D.; Kim, M. R.; Sok, D. E. Oral Administration of 2Docosahexaenoyl Lysophosphatidylcholine Displayed Anti-Inflammatory Effects on Zymosan A-Induced Peritonitis. Inflammation 2011, 34, 147-160.

(36) Yamashita, S.; Fujiwara, K.; Tominaga, Y.; Nguma, E.; Takahashi, T.; Otoki, Y.; Yamamoto, A.; Higuchi, O.; Nakagawa, K.; Kinoshita, M.; Miyazawa, T. Absorption Kinetics of Ethanolamine Plasmalogen and Its Hydrolysate in Mice. J. Oleo Sci. 2021, DOI: $10.5650 /$ jos.ess20223.

(37) Yamashita, S.; Honjo, A.; Aruga, M.; Nakagawa, K.; Miyazawa, T. Preparation of Marine Plasmalogen and Selective Identification of Molecular Species by LC-MS/MS. J. Oleo Sci. 2014, 63, 423-430.

(38) Yamashita, S.; Kanno, S.; Nakagawa, K.; Kinoshita, M.; Miyazawa, T. Extrinsic Plasmalogens Suppress Neuronal Apoptosis in Mouse Neuroblastoma Neuro-2A Cells: Importance of Plasmalogen Molecular Species. RSC Adv. 2015, 5, 61012-61020.

(39) Harada-Shiba, M.; Kinoshita, M.; Kaimado, H.; Shimokado, K. Oxidized Low Density Lipoprotein Induces Apoptosis in Cultured Human Umbilical Vein Endothelial Cells by Common and Unique Mechanisms. J. Biol. Chem. 1998, 273, 9681-9687.

(40) Narayanan, B. A.; Narayanan, N. K.; Desai, D.; Pittman, B.; Reddy, B. S. Effects of a Combination of Docosahexaenoic Acid and 1,4-Phenylene Bis(Methylene) Selenocyanate on Cyclooxygenase 2, 
Inducible Nitric Oxide Synthase and $\beta$-Catenin Pathways in Colon Cancer Cells. Carcinogenesis 2004, 25, 2443-2449.

(41) Yamashita, S.; Seino, T.; Aida, K.; Kinoshita, M. Effects of Plant Sphingolipids on Inflammatory Stress in Differentiated Caco-2 Cells. J. Oleo Sci. 2017, 66, 1337-1342.

(42) Kinoshita, M.; Shimokado, K. Autocrine FGF-2 Is Responsible for the Cell Density - Dependent Susceptibility to Apoptosis of HUVEC; A Role of a Calpain Inhibitor - Sensitive Mechanism. Arter. Thromb Vasc Biol. 1999, 19, 2323-2329.

(43) Folch, J.; Lees, M.; Stanley, G. H. S. A Simple Method for the Isolation and Purification of Total Lipides from Animal Tissues. J. Biol. Chem 1957, 226, 497-509.

(44) Yamashita, S.; Shimada, K.; Sakurai, R.; Yasuda, N.; Oikawa, N.; Kamiyoshihara, R.; Otoki, Y.; Nakagawa, K.; Miyazawa, T.; Kinoshita, M. Decrease in Intramuscular Levels of Phosphatidylethanolamine Bearing Arachidonic Acid during Postmortem Aging Depends on Meat Cuts and Breed. Eur. J. Lipid Sci. Technol. 2019, 121,1800370 .

(45) Otoki, Y.; Kato, S.; Kimura, F.; Furukawa, K.; Yamashita, S.; Arai, H.; Miyazawa, T.; Nakagawa, K. Accurate Quantitation of Choline and Ethanolamine Plasmalogen Molecular Species in Human Plasma by Liquid Chromatography-Tandem Mass Spectrometry. J. Pharm. Biomed. Anal. 2017, 134, 77-85. 